\title{
DYNAMIC CHANGE OF VIRTUAL TIME WHEN MODELING VEHICLE OPERATING CYCLES IN THE PROGRAM 'STAMM'
}

\author{
A.V. MANYASHIN \\ Tyumen Industrial University, Russia
}

\begin{abstract}
The article explores the problem of modelling performance properties of cars in real driving conditions. At the same time, real or synthetic driving cycles are used to reproduce the road situation in the simulation model. The composition, the ratio of individual components and the nature of speed changes in such cycles significantly depends on both the traffic conditions on the route and the design features of the vehicles.

The purpose of this work is to optimize simulation models of car operation by reducing the time of the experiment while maintaining the required accuracy of the model.

This result is achieved using a dynamically changing model time. The paper considers the features of driving cycles of cars for various purposes. The program of simulation modelling Stamm 4.1 is presented, which implements the method of dynamic change of model time when simulating driving cycles of cars, which allows significantly reducing the duration of the experiment.

Keywords: operating cycle of a vehicle, simulation modelling, urban traffic conditions.
\end{abstract}

\section{INTRODUCTION}

A car is a system containing a large number of elements that have complex relationships with each other. At the same time, it is itself part of a complex driver - car-road - environment system. Therefore, the process of changing the speed of the car during its operation is not stationary. This is especially noticeable in urban settings, where the speed at any given time is determined by the interaction with other road users. Meanwhile, the speed of the car is the most important factor determining the so-called mode of operation of the car. Therefore, it is very important to accurately reproduce the vehicle's speed profile when computer simulating vehicle operating modes.

It is known that some parameters of the car change randomly while driving, under the influence of a large number of external factors. For example, the speed of a car and its associated parameters depend on the road situation, road surface condition, weather conditions, driving style and other factors. On the other hand, if the vehicle is idle, most processes are stationary, and their parameters change rather slowly. If we consider the operation of the car for a fairly long period of time, we can see that individual phases with different intensity of speed changes are cyclically repeated. Therefore, to reproduce the operation of the car, for example, for the purpose of rationing fuel consumption or emissions of harmful substances with exhaust gases, typical driving cycles are often used. And these tests themselves are often carried out using computer simulations. However, typical driving cycles for vehicles that perform special functions are usually not used.

Based on the above, the simulation of car operation is usually carried out in the form of a simulation experiment. Typical driving cycles are usually used in these models to reproduce the speed profile that corresponds to the intended operating conditions of vehicles. Usually, there are driving cycles that correspond to the type of vehicle and the prospective area of use of the car. For example, urban, extra-urban, for buses, cars and trucks. However, there is not a large variety of typical cycles for special vehicles, although it is not difficult to assume 
that they will differ significantly from similar profiles for universal vehicles, due to the need to perform special functions first. In addition, it is often necessary to simulate the processes that occur not only when the car is moving, but also during its downtime or parking. This is possible if you use the appropriate operating cycles, and when reproducing them in the simulation model, take into account the different intensity of parameter changes in different phases of the cycle.

\section{THEORETICAL JUSTIFICATION FOR THE USE OF TYPICAL OPERATING CYCLES OF DIFFERENT TYPES}

All the typical driving cycles known in the world represent the dependence of speed on time or distance travelled, i.e. speed profiles. The main purpose of typical driving cycles is to correspond as accurately as possible to real speed profiles that correspond to certain operating conditions. Therefore, it is quite obvious that the type and content of such a cycle will significantly depend on the conditions in which the car is supposed to be used and what additional functions it should perform, for example, stop according to the schedule, if it is a bus. If we consider the actual driving cycles of a universal (Fig. 1) and a special car (Fig. 2), recorded at the same time and on the same road network, we can see that they differ significantly.

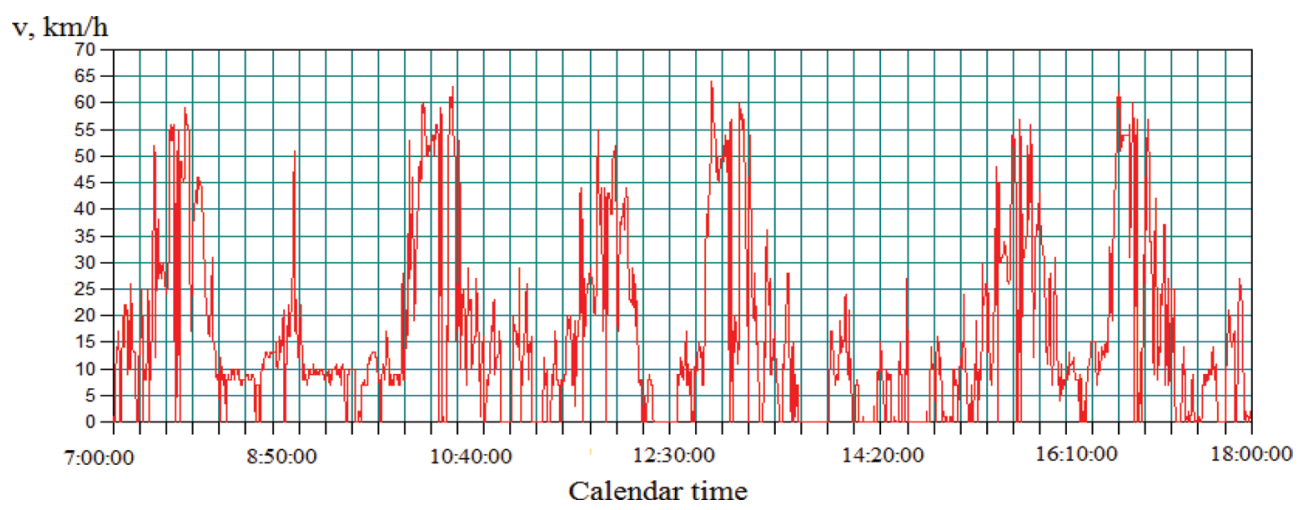

Figure 1: Driving cycle of a universal car.

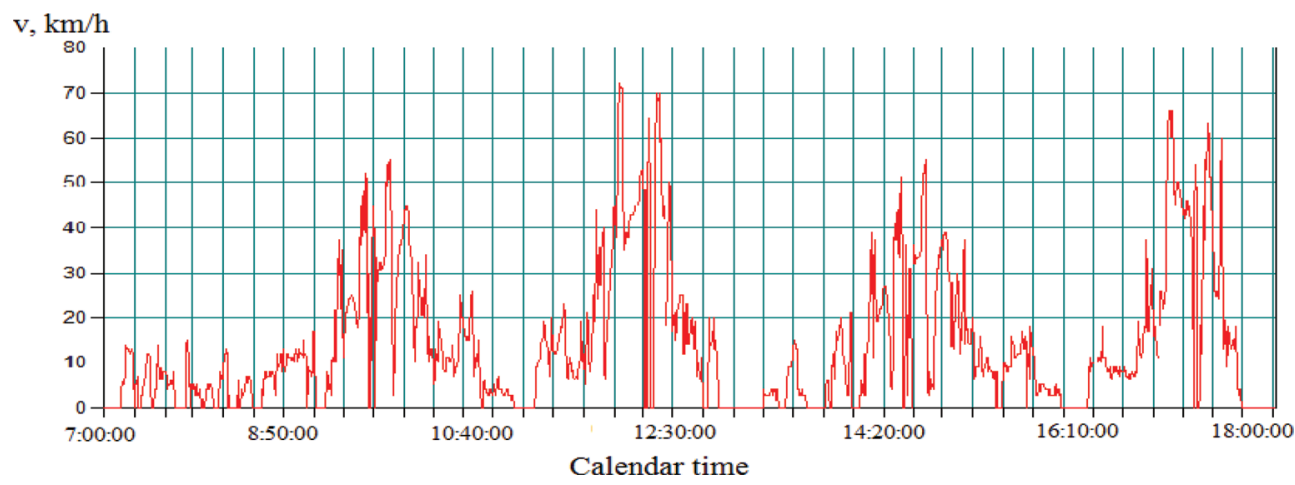

Figure 2: Driving cycle of concrete mixer trucks. 


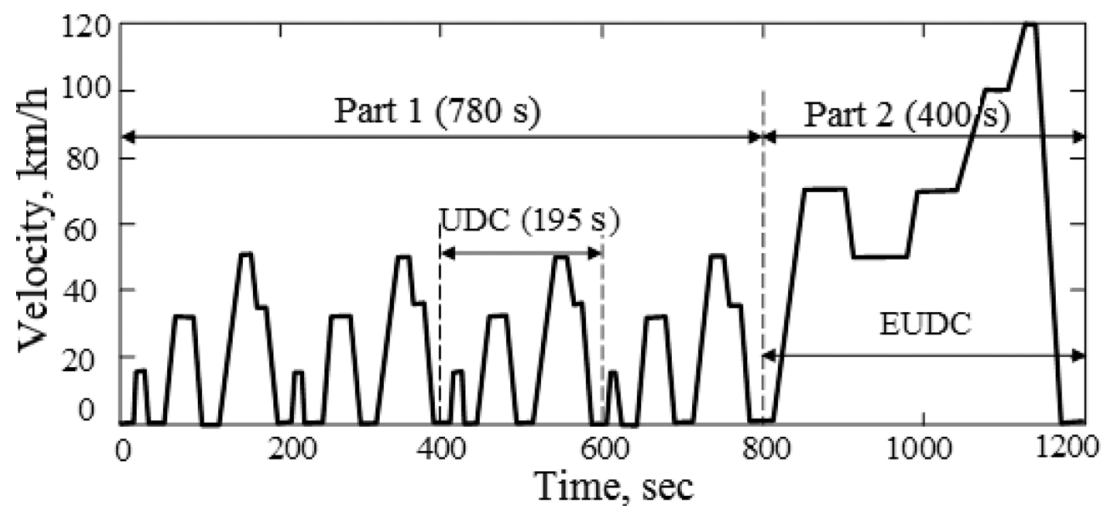

Figure 3: New European driving cycle [2].

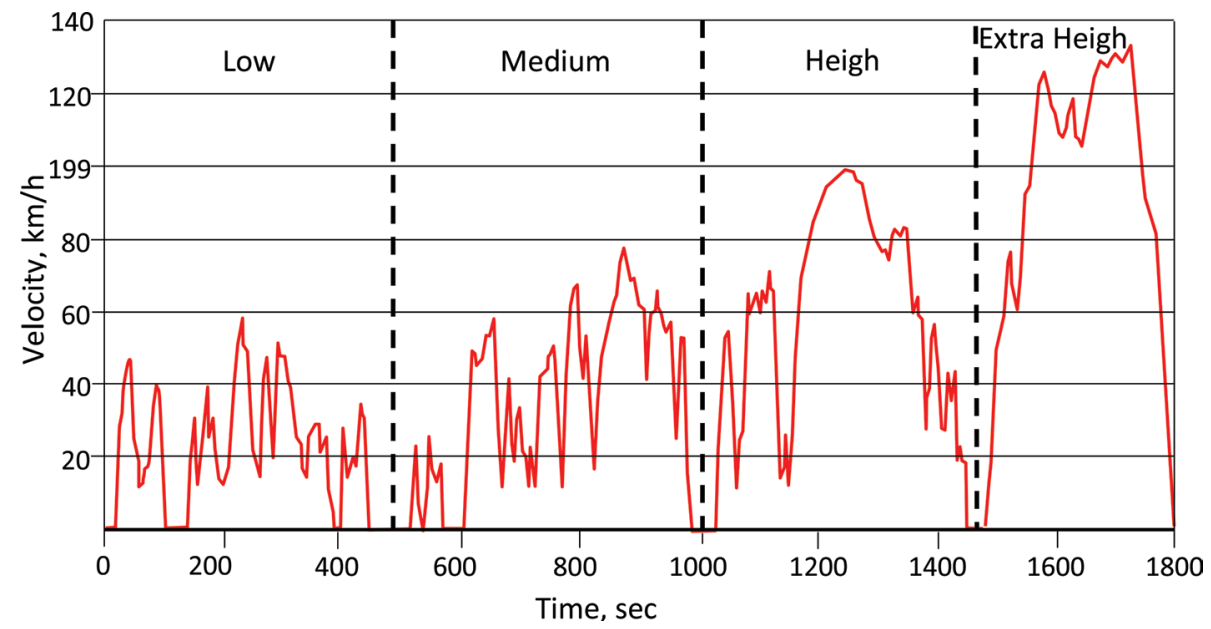

Figure 4: Driving cycle WLTC [7].

This fact must be taken into account when modelling processes and systems of the car. Very often, so-called modal driving cycles are used when there are sections of traffic with a constant value of speed or acceleration of the car, for example, the European driving cycle NEDC (new European driving cycle) [2] (Fig. 3). The NEDC speed profile can be easily reproduced in simulation models using simple analytical equations. However, such cycles do not accurately reflect the actual speed profiles of cars (see Figs. 1 and 2) by 2017, the world driving cycle has been developed [7] (Fig. 4). If you use WLTC to model the movement of a car, simple mathematical dependencies are no longer sufficient and you need to use other methods of data representation.

2.1 Influence specialization of a car on the features of its operation

Analysis of the ratio of the duration of various operations performed by different types of vehicles during the day shows significant differences (Fig. 5). For example, cranes 
Hours

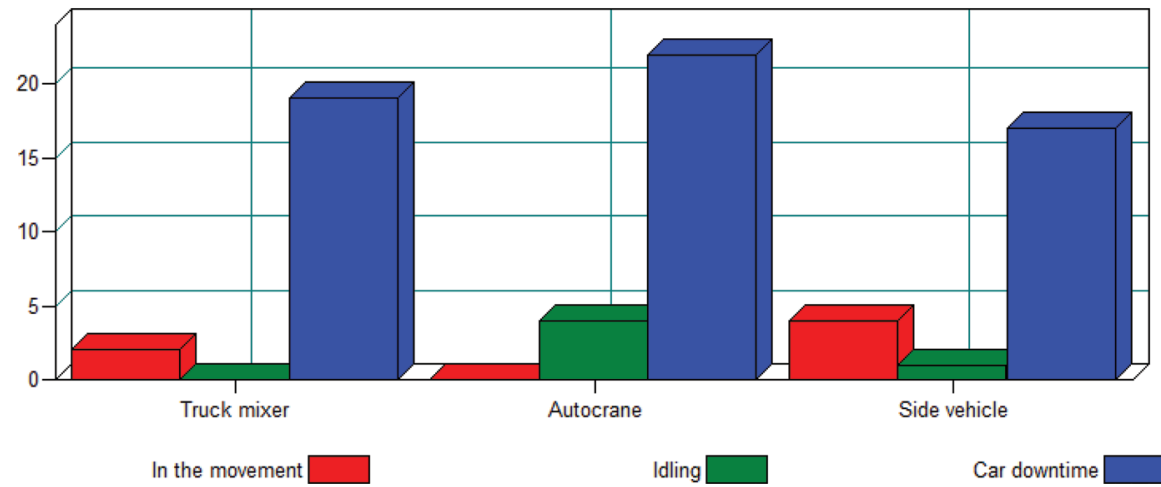

Figure 5: The ratio of operating modes during the day for different types of vehicles.

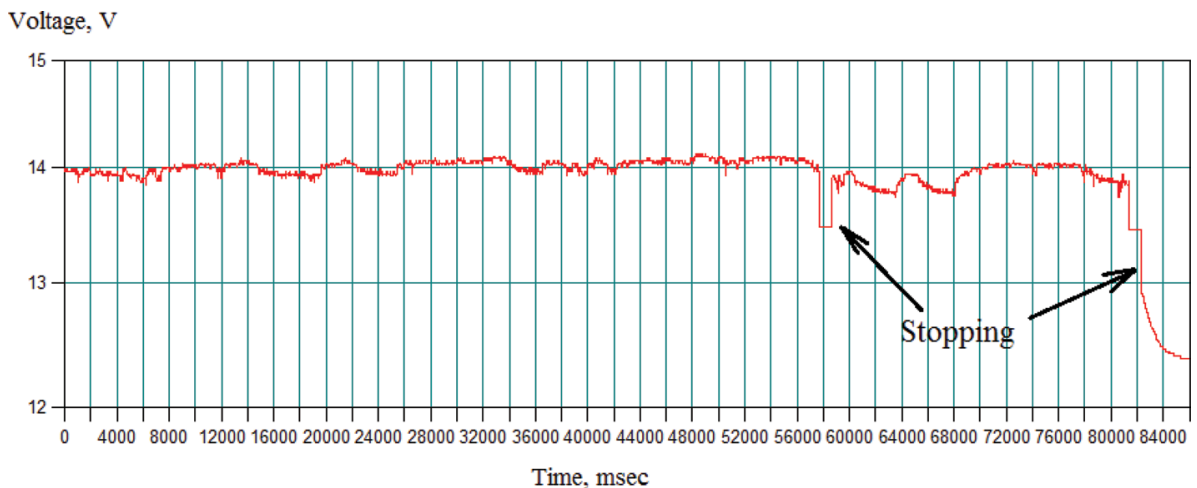

Figure 6: Battery voltage when driving a car.

perform the main work during parking, so the part of the time that falls on the movement of the car is minimal. Truck mixers, on the contrary, perform the main job of mixing the mixture while driving, so the engine of such transport units practically does not work at idle.

\subsection{Daily vehicle operating cycles}

Very often, there is a need to study long-term processes, the parameters of which have different speeds during driving and during stopping or parking the car. For example, the ambient temperature or car battery voltage (Fig. 6).

In such cases, instead of the driving cycle, it is advisable to use daily operating cycles, which will differ significantly for vehicles of different purposes (Fig. 7). 


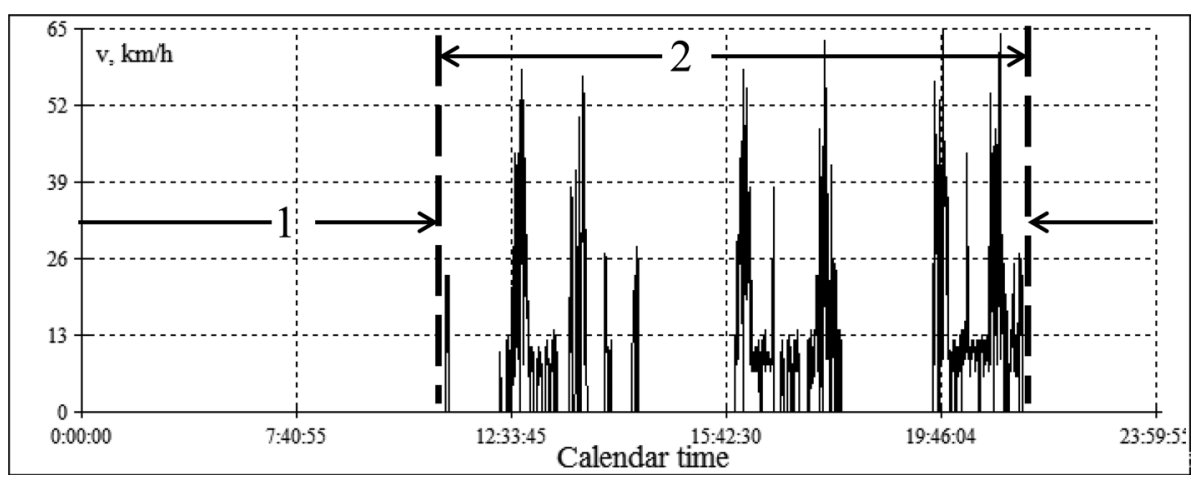

Figure 7: Velocity profile of the universal car during the shift: (1) idle and (2) movement.

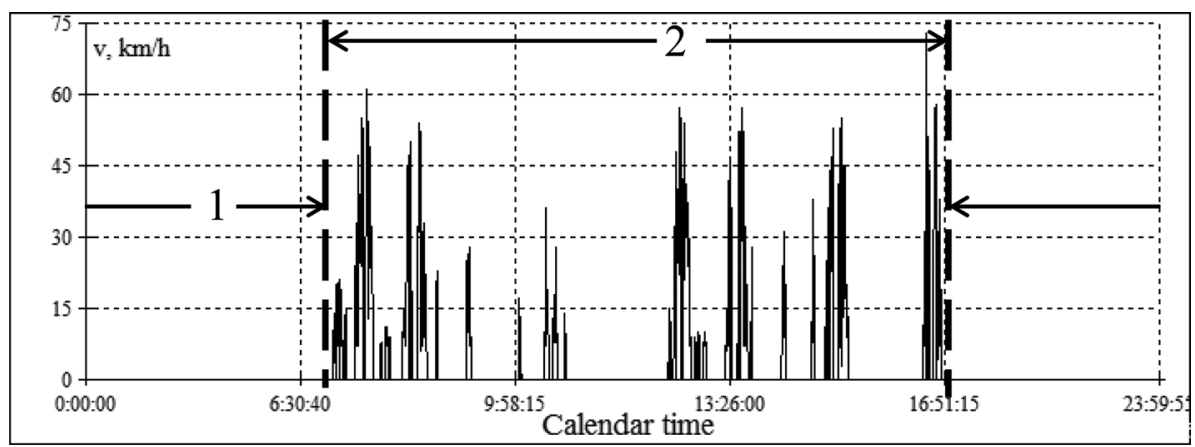

Figure 8: Velocity profile of the special car (mixer): (1) idle and (2) movement.

\section{OPTIMIZATION OF THE VIRTUAL TIME STEP OF STAMM SIMULATION MODELS}

The author of this work prepared the program 'Stamm' for the initial processing of experimental data and organization of system dynamics models based on spreadsheets [5] (Fig. 9). A distinctive feature of the application is its small size and portability, in fact, it is a single software module of about $8 \mathrm{MB}$ in size. On the basis of 'Stamm', research was conducted in the field of road transport, which formed the basis of several dissertations [1, 2]. The system is a non-commercial product and is used in research and educational organizations. The program's working files are workbooks, originally consisting of spreadsheets similar to Microsoft's Excel.

As the program was improved, it received new functions necessary for scientific research in the field of transport. In version 2.0, data visualization is now possible, and workbooks consist of different types of sheets. Stamm 3.0 can perform frequency analysis of data using Fourier and Wavelets transforms. Also, components have been added to calculate the parameters of the multivariate regression by the method Levenberg-Marquardt. Version 3.0 also gained the ability to cluster data analysis and create simple neural networks. Finally, in version 4.0, it is possible to visually design components of simulation models and create models of system dynamics, agent-based and mixed models on this basis. In addition, to ensure compatibility between future versions of the program, it is possible to save workbooks in XML format in the latest version. 
The possibilities of organizing simulation modelling in the program are represented by using two types of models. The first type is table models, which consist of a two-dimensional sheet similar to Microsoft Excel, but the cells of this sheet can use cross-references, scan table data in other workbook sheets, and manage model time. Thus, it is possible to create simulation models of system dynamics. The second type is object visual models. Both types support dynamically changing the simulation step during the simulation.

Whatever type of computer simulation is used, the time change in the models occurs discretely with a certain step, the minimum value of this step is determined by the processor clock frequency. The Stamm program sets the numerical value of the model time increment using a special internal variable 'Step'. This variable is available for modification in model elements. This makes it possible to model the operating cycles of cars taking into account the characteristics of individual phases (Fig. 10).

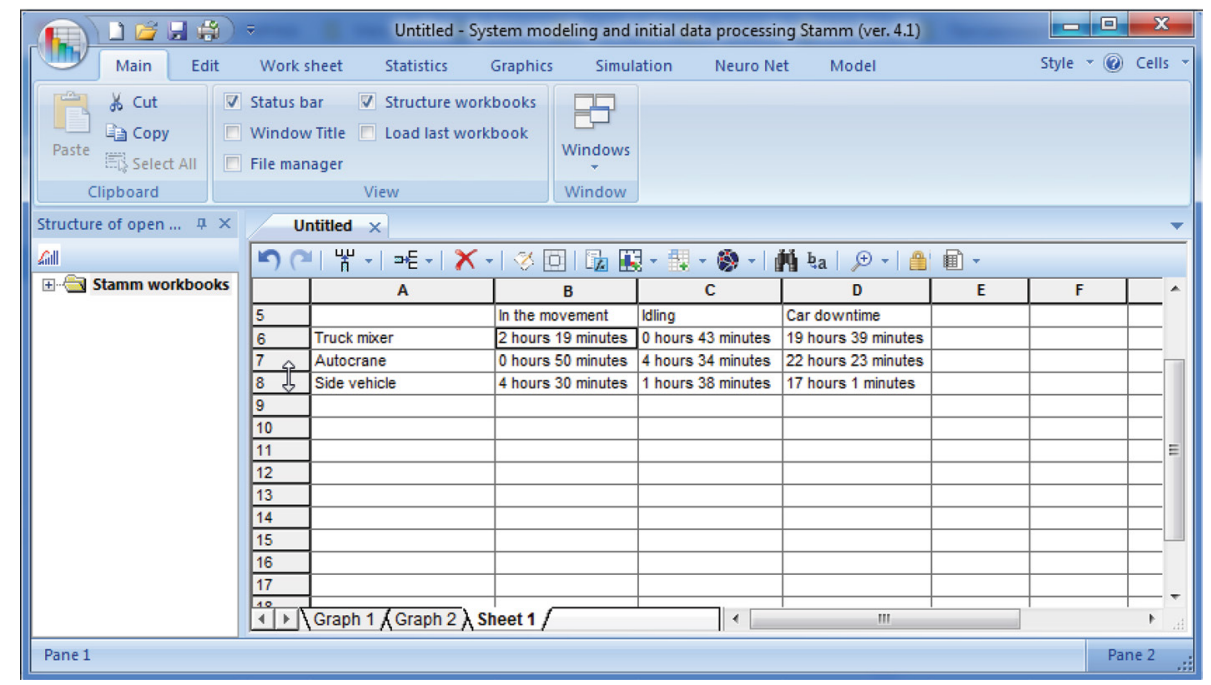

Figure 9: Program ‘Stamm’ version 4.1.

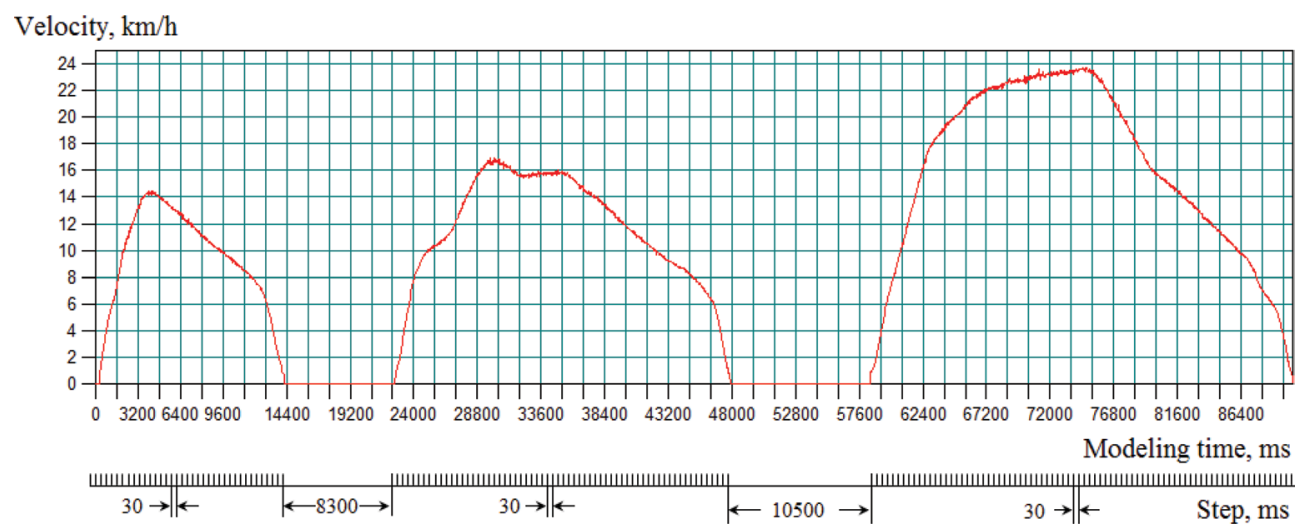

Figure 10: Dynamically changing the model time step when simulating the vehicle's operating cycle. 
The data are shown in Fig. 10 this is a part of the operating cycle model that corresponds to the simulation of the fuel consumption process when driving a car in urban conditions with the engine fully warmed up. There are two stops with the engine idling. In both of these cases, the model time changes abruptly in accordance with the cycle profile by the amount of the stop time. When reproducing the process of driving a car in the model, the increment step of the virtual time is $30 \mathrm{~ms}$.

\subsection{Dynamic change of model time discreteness in table-type models}

In a table-type model, the value of the modelling step is set in the settings panel (Fig. 11) - the 'Step' field. In this case, you can set the initial numerical value of the step in the corresponding field. If you need to dynamically change the step during the simulation experiment, in the simulation parameters settings panel (Fig. 10), in the 'Synchronize by' field, you must specify the address of the cell that will contain the current step value. The value of this cell, as well as all the others, can change dynamically in accordance with the model logic, and the increment of the model time will change accordingly.

The value of the increment step of the model time changes during the playback of a tabletype model in a special controller cell in accordance with the specified expression (Fig. 12).

For example, the values of the simulation step can be changed depending on the current phase of the operational cycle reproduced in the model (highlighted in red rectangle in Fig. 13). in this case, you can simulate the phases of the daily operational cycle of ground vehicles with different increments of time.

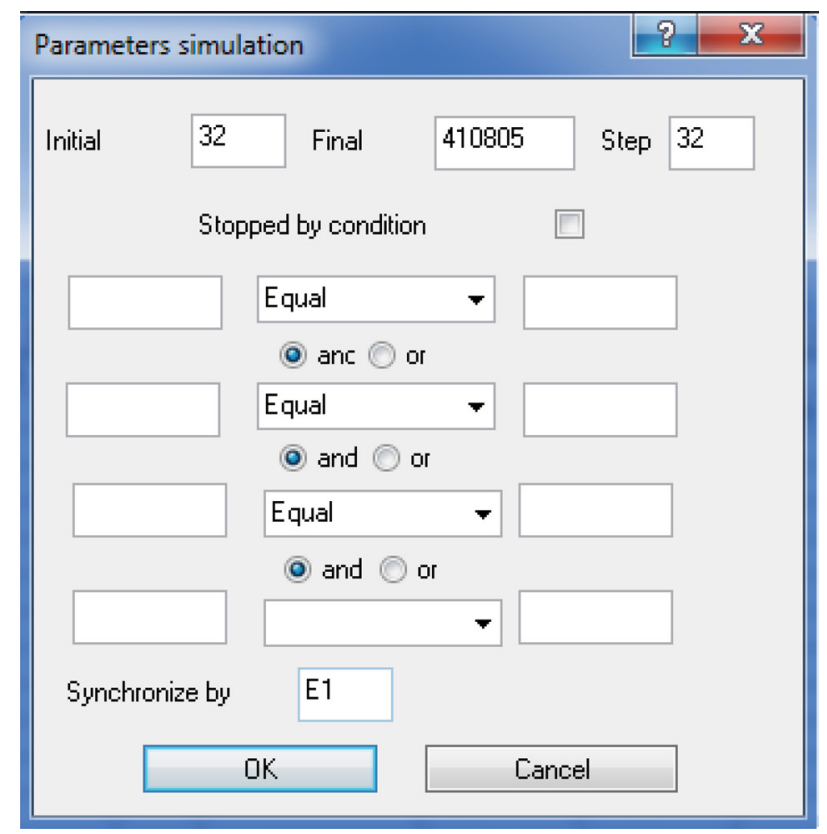

Figure 11: The window for setting parameters for simulating a table-type model. 


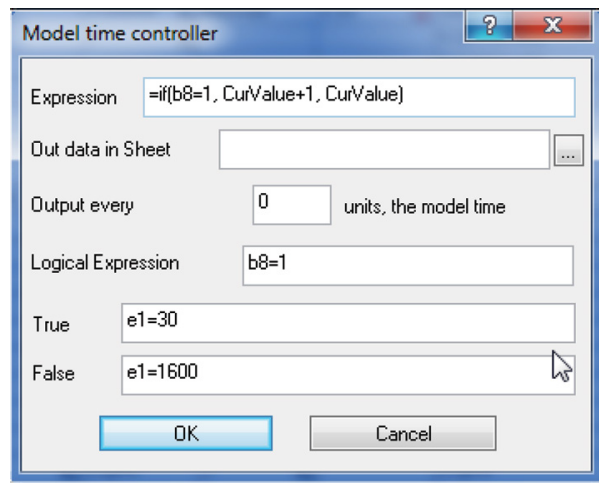

Figure 12: Panel for setting parameters cell-controller of a table-type model.

\begin{tabular}{|c|c|c|c|c|c|c|c|c|c|c|}
\hline \multicolumn{11}{|c|}{ Fuel consumption.swb $x$} \\
\hline \multicolumn{11}{|c|}{ ด } \\
\hline & A & B & c & $\mathrm{D}$ & $E$ & $\mathrm{~F}$ & G & $\mathrm{H}$ & 1 & $\mathrm{~J}$ \\
\hline 1 & Vehicle & & & Step & 10 & Cycle time, ms & $f_{x}$ & 0 & $\min$ & \\
\hline 2 & GAZ-32213 & & Useful loading & & & Speed & 庵 & $f_{*}$ & $\mathrm{~km} / \mathrm{h}$ & \\
\hline 3 & Weight & 3500 & 1000 & & & Acceleration & $f_{x}$ & $\mathrm{~m} / \mathrm{s} 2$ & & \\
\hline 4 & The radius of the whe & 0.37 & & & & Previous value & 0 & & & \\
\hline 5 & Gear ratio of a transmi & i 5.6 & & & & & & & & \\
\hline 6 & Height & 2075 & & & & & & & & \\
\hline 7 & Width & 2200 & & & & & & & & \\
\hline 8 & Kobt & 0.65 & & & & & Nk & 0 & W & \\
\hline 9 & Transmission efficienc & 0.92 & & & & & $\mathrm{Ne}$ & 0 & W & \\
\hline 10 & Maximum speed & 120 & & & & & $\mathrm{Nj}$ & 0 & W & \\
\hline 11 & Fuel consumption at 9 & & & & & Correction & ko6 & $f_{x}$ & & \\
\hline \multicolumn{11}{|l|}{12} \\
\hline \multicolumn{11}{|l|}{13} \\
\hline 14 & Engine & ZMZ-4062.1 & & & & & Run & 0 & $m$ & \\
\hline 15 & Specific effective fuel & 265.2 & Revolutions idle & 800 & & & Hourly con & $1.6505791 f_{x}$ & Uh & \\
\hline 16 & Fuel density & 0.74 & Consumption on idle & $1.6 / 579150$ & Vhour & & General & $f_{x}$ & liter & \\
\hline 17 & Internal combustion en & 2280 & Coef. revolutions & 15 & & & Specific & 0 & $V 100 \mathrm{~km}$ & \\
\hline 18 & $\begin{array}{l}\text { Number nf mulinders } \\
\text { GAZ-32213 \& Graph }\end{array}$ & 4 Urban $c y$ & 1 & & 1 & & & III & & \\
\hline
\end{tabular}

Figure 13: Stamm worksheet of the type 'simulation model'.

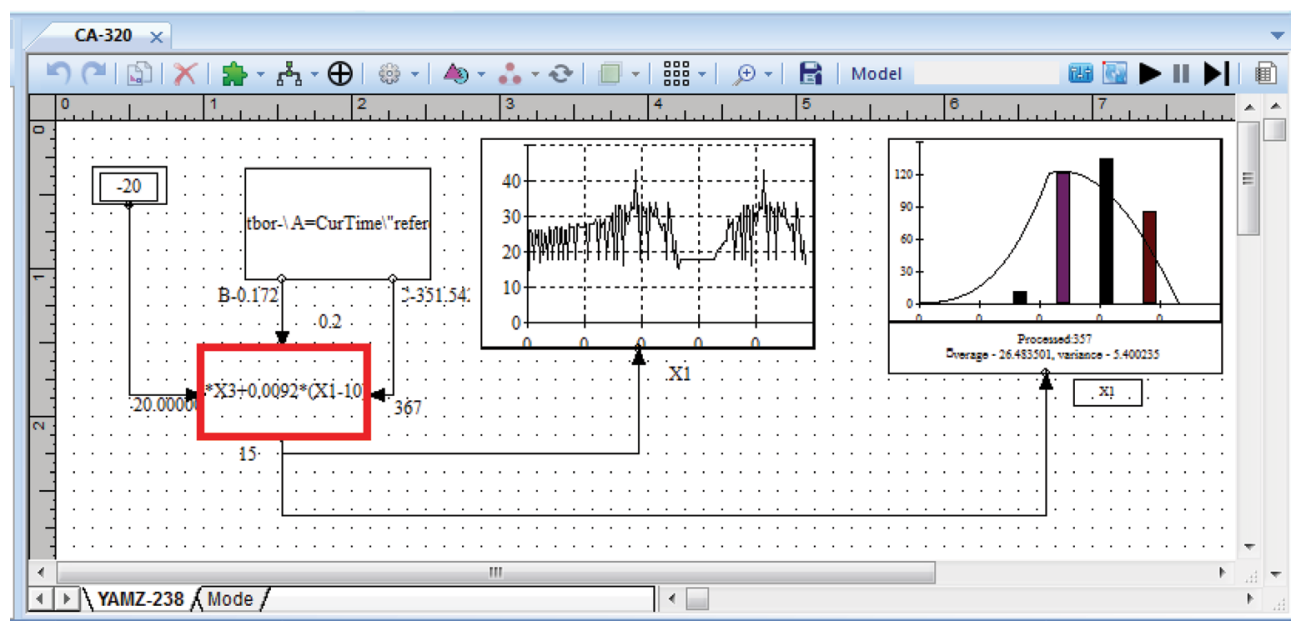

Figure 14: Object model. The Component 'Formula' 


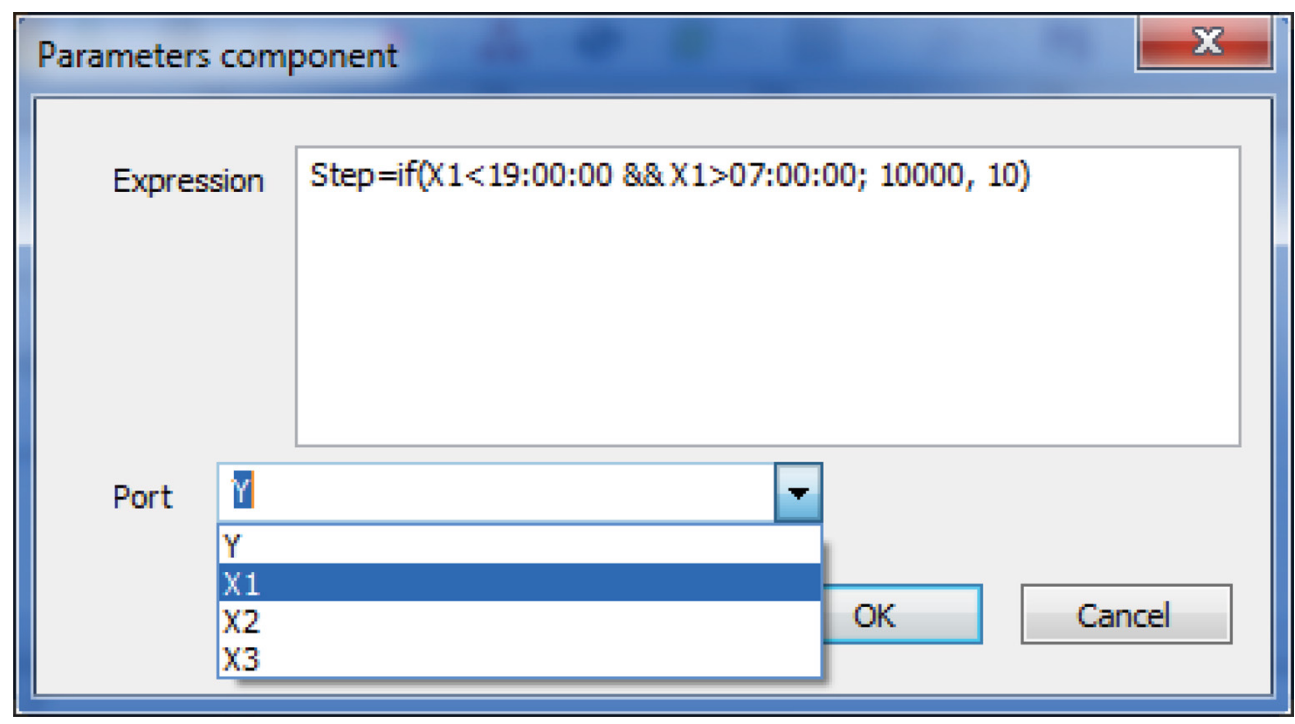

Figure 15: Dynamically changing the modelling step in the settings panel of the visual component of the type 'Formula'.

\subsection{Variable step of modelling in object models}

When an object visual simulation model is used, the dynamic change of the model time step occurs in special visual components - 'Formula' (highlighted in red rectangle in Fig. 14).

To dynamically change the model time increment, put a logical expression containing this variable in the component (Fig. 15).

\section{CONCLUSION}

Summing up, it is possible to formulate the main recommendations for the organization of simulation models of the processes changing the operational properties of special vehicles during their technological and transport operations.

- The speed and load profiles of vehicles performing special functions differ significantly from similar curves for universal vehicles. The nature of the change in the load on the engine and the speed of a special car during its operation is mainly determined by the peculiarity of its technological operations. For example, the operating cycles of cars that perform special functions during movement and during parking are radically different.

- Curves of speed changes in real driving cycles of cars cannot be reconstructed using simple analytical dependencies, it is advisable to use discrete values in the form of tabular functions.

- Different phases of the full operational cycle of a special vehicle cause different rates of change in operational parameters, such as speed, engine crankshaft speed, the temperature of components and assemblies, fuel consumption, etc.

- When simulating the operation of special vehicles, it is necessary to take into account the above features. For adequate reproduction of continuous processes in the model using discrete table functions, it is important to choose the optimal increment step of the model 
time. So, based on the above features, this step should be different and change depending on the correspondence of the current model time to a particular phase of the cycle.

- Unlike well-known modelling systems that use adaptive [3, 4, 8] change of the model time step and calculate its value before performing the next step, the Stamm program changes the value of the 'Step' variable depending on a logical condition or with reference to a specific value of the current model time.

The Stamm system considered in this paper, which provides an adaptive change in the virtual computer time step during a simulation experiment, can be used to simulate the operating cycles of universal or special vehicles. Future research provides for obtaining typical operating cycles of special vehicles for various purposes.

\section{REFERENCES}

[1] Karnaukhov, N.N., Reznik, L.G. \& Manyashin, A.W., The influence of automobiles motion unevenness on harmful substances emission. Proceedings of the Eighth International Conference on Urban Transport and the Environment in the 21st Century, pp. 553-560, 2002.

[2] Manyashin, A.V. \& Manyashin, S.A., Method for synthesizing the driving cycle of a car. International Scientific Journal, 1, pp. 87-91, 2012.

[3] Marks, N. \& Robinson, M., Variable timestep algorithm for molecular dynamics simulation of non-equilibrium processes. Nuclear Instruments and Methods in Physics Research, Section B: Beam Interactions with Materials and Atoms, 352, pp. 3-8, 2015.

[4] Nzale, W. \& Mahseredjian J., Two Variable Time-Step Algorithms for Simulation of Transients, IEEE Milan PowerTech, pp. 3076-3082, 2019.

[5] Manyashin, A., Simulation of transport operation in urban conditions with variable virtual time increments in the program Stamm 4.1, WIT Transactions on the Built Environment, vol. 200, WIT Press: Southampton and Boston, pp. 185-192, 2020.

[6] The program for initial data processing, creating simulation models and data visualization. [Online]. https://www.stamm.uno (accessed 19 February 2021).

[7] The Worldwide Harmonised Light Vehicle Test Procedure (WLTP). [Online]. https:// www.vehicle-certification-agency.gov.uk/fcb/wltp.asp (accessed 22 February 2021).

[8] Variable Time step in Transient simulation. Ansys Learning Forum. [Online]. https:// forum.ansys.com/discussion/7118/variable-time-step-in-transient-simulation (accessed 4 January 2021). 